\title{
Comportement reproductif des porcs de race Duroc en République du Congo
}

\author{
Alexis Jonas OGNIKA ${ }^{1 *}$, Parisse AKOUANGO ${ }^{1}$, Annick Raïssa EBENGO GNANGA ${ }^{1}$ et \\ Silvère DIMI NGATSE ${ }^{2}$
}

\author{
${ }^{1}$ Laboratoire des Productions Animales et Biodiversité, École Nationale Supérieure d'Agronomie et de \\ Foresterie, Université Marien Ngouabi, Congo. BP. 69 Brazzaville, Congo. \\ ${ }^{2}$ École Nationale Supérieure d'Agronomie et de Foresterie, Université Marien Ngouabi, Congo. \\ Tél : (00242)066698519) \\ *Auteur correspondant ; E-mail: alexisjonaso@gmail.com; Tél: (+242)069642961 /050370789
}

Received: 13-03-2021 Accepted: 05-10-2021 $\quad$ Published: 30-10-2021

\section{RESUME}

La présente étude a été menée dans le cadre de l'amélioration des performances de reproduction et de croissance des porcs locaux par l'introduction de nouvelles races. Il s'agit d'une préoccupation des politiques du Gouvernement congolais. L'objectif de l'étude a été d'apprécier certains paramètres de reproduction et de croissance des porcs Duroc dans le but de maitriser leurs aptitudes en vue de les utiliser dans le programme d'amélioration génétique du porc local congolais. Les animaux avaient huit (8) mois d'âge. L'évaluation des caractéristiques de reproduction et de croissance a concerné 10 femelles, 5 mâles en stade de reproduction et 145 porcelets. Les caractéristiques reproductives et de croissance ont été évaluées selon une méthode de pointage de 1 à 5 . Les productivités numérique et pondérale ainsi que le rendement carcasse ont également été estimés. La taille de la portée par truie est de $9 \pm 0,8$ têtes, le poids de la portée par truie est de $25,08 \pm 8 \mathrm{~kg}$ et l'âge de mise en reproduction est de $8,3 \pm 0,7 /$ mois. Les animaux ont indiqué de bonnes aptitudes de reproduction. Le poids vif moyen des porcelets est de $1,32 \pm 0,4 \mathrm{~kg}$ à la naissance, de $9,99 \pm 0,1 \mathrm{~kg}$ la $4{ }^{\text {ème }}$ semaine correspondant au sevrage et de $50,4 \pm 6,5 \mathrm{~kg}$ la $16^{\text {ème }}$ semaine. Les GMQ ont été respectivement de $230 \pm 0,5 \mathrm{~g}$ la $2^{\text {ème }}$ semaine, $370 \pm 0,3 \mathrm{~g}$ la $4^{\text {ème }}$ semaine et de $650 \pm 0,8 \mathrm{~g}$ la $16^{\text {ème }}$ semaine. Ces indicateurs augurent de bonnes perspectives de croissance des animaux de la race en République du Congo.

(C) 2021 International Formulae Group. All rights reserved.

Mots clés : Duroc, reproduction, porc, Congo.

\section{Reproductive behavior of Duroc pigs in the Republic of Congo}

\begin{abstract}
This study was carried out in the context of improving the reproductive and growth performance of local pigs by introducing the new races. It concerns the Congolese government programs. The objective of this study was to appreciate some reproduction and growth parameters of Duroc pigs with the aim to master their aptitudes for to use them in the genetic improvement program of the local Congolese pig. The animals were eight (8) months old. The reproductive evaluation and growth characteristics concerned 10 females, 5 males in the reproductive stage and 145 piglets. Reproductive and growth characteristics were assessed using a 1 to 5 scoring method. The numerical and weight productivities and the carcass yield were also estimated. The litter size per
\end{abstract}


sow is $9 \pm 0.8$ head, litter weight per sow is $25.08 \pm 8 \mathrm{~kg}$ and breeding age is $8.3 \pm 0.7 /$ month. The animals indicated good breeding skills. The average live weight of piglets is $1.32 \pm 0.4 \mathrm{~kg}$ at birth, $9.99 \pm 0.1 \mathrm{~kg}$ in the 4 th week corresponding to weaning and $50.4 \pm 6.5 \mathrm{~kg}$ in the 16 th week. The ADGs were respectively $230 \pm 0.5 \mathrm{~g}$ in the 2 nd week, $370 \pm 0.3 \mathrm{~g}$ in the 4 th week and $650 \pm 0.8 \mathrm{~g}$ in the 16 th week. These indicators bode well for growth prospects for animals of the breed in the Republic of Congo.

(C) 2021 International Formulae Group. All rights reserved.

Keywords: Duroc, reproduction, pig, Congo.

\section{INTRODUCTION}

La révolution agricole dans le monde occidental a permis une réduction de la charge de travail et une augmentation de la production agricole (Kimsé et al., 2013). En Afrique, l'agriculture au sens large connaît un début de mécanisation et ne satisfait pas encore les besoins de sa population. Au Congo, la structure de l'économie actuelle basée sur l'exploitation des ressources non renouvelables fait appel au développement de nouveaux secteurs capables de porter l'économie afin de favoriser une meilleure redistribution de la richesse. L'élevage, l'un des nouveaux secteurs occupe une place importante dans le développement du pays (Mopoundza et al., 2019).

En République du Congo, l'analyse des principales filières de l'élevage montre que l'ensemble de la production ne couvre que très faiblement les besoins de consommation des populations. (Ognika et al., 2016). Aussi, les données du sous-secteur porcin révèlent que la consommation de la viande de porc en 2011 a été de 7,359 tonnes, sur la base d'un taux d'accroissement annuel de la population de l'ordre de $3 \%$, le besoin en viande porcine en 2016 était estimé à 13694,4 tonnes, soit une hausse de 86,09 \%.(DGE, 2014).

En effet, le porc est un excellent assimilateur ( Accodji et al.,2009). Mais, le problème de l'élevage porcin congolais réside dans le fait que les dernières importations officielles des races améliorées remontent aux années soixante-dix et les mises en reproduction des animaux ont été faites avec les races disponibles dans le pays. Toutefois, l'importation des porcs de race Duroc en 2012 s'est faite dans le but d'améliorer les performances de la race locale (Ognika, 2018).
Le Duroc est une race porcine originaire des Etats-Unis d'Amérique où il est apparu au cours du XIX $^{\mathrm{e}}$ siècle à la suite de divers croisements opérés entre des cochons à la robe rouge. Il appartient à la famille des sus scrofa domesticus souvent utilisé dans les croisements industriels et comme verrat terminaux ( Ifip, 2018).

Ainsi, l'étude de la race Duroc assure la connaissance de son niveau de performance dans le pays et contribuera assurément à garantir le renouvellement de la race et augmenter la productivité des porcs locaux par le biais des croisements. Dans cet ordre, l'objectif de cette étude est de caractériser les performances reproductives des animaux de race Duroc élevés en République du Congo.

\section{MATERIEL ET METHODES}

\section{Situation géographique}

La présente étude s'est réalisée dans le Centre de Vulgarisation des Techniques d'Elevage (CVTE) de Kombé situé à environ $17 \mathrm{~km}$ au sud de Brazzaville. Il est spécialisé dans la production des reproducteurs porcins et la vente de la viande porcine à destination du marché local. La zone d'étude est située dans la partie Ouest de la zone équatoriale entre le $4^{\circ}$ degré de latitude nord et le $5^{\circ}$ degré de latitude sud et le $11^{\circ}$ de longitude Est et le $19^{\circ}$ de longitude Ouest.

\section{Caractéristiques climatiques}

La zone d'étude se situe dans le secteur climatique bas congolais marqué par une pluviométrie relativement abondante avec des précipitations comprises entre 800 et 1800 $\mathrm{mm}$, une variabilité Interannuelle de 15 à $40 \%$. Dans cette zone, la saison sèche est presque absolue de 150 à 160 jours de mai à septembre. Une saison des pluies d'octobre à mai avec des 
fléchissements intra-pluvial en janvier février.

Dans ce département, les températures moyennes annuelles se situent entre 21 et $27^{\circ} \mathrm{C}$ avec des écarts annuels de 4 à $5{ }^{\circ} \mathrm{C}$ et diurnes de 6 à $11^{\circ} \mathrm{C}$. L'humidité relative est de 80 à $87 \%$ avec de faibles variations saisonnières.

\section{Caractéristiques hydrographiques}

Le réseau hydrographique de la zone d'étude est dense. Il s'organise autour du principal bassin du Congo, auquel s'ajoutent des petits affluents. Seulement $7 \%$ de la superficie totale du bassin du fleuve Congo (soit $230000 \mathrm{~km}^{2}$ ) sont situés sur le territoire congolais. Son débit interannuel de 41.700 $\mathrm{m}^{3} / \mathrm{s}$ lui confère le rang de deuxième fleuve du monde après l'Amazone.

\section{Caractéristiques pédologiques}

Le relief de la zone d'étude est très varié. II est caractérisé par des zones de collines, de plateaux et de montagnes de moyenne altitude. Les sols sont le plus souvent ferralitiques, très fragiles, acides et sensibles aux érosions hydriques et/ou éoliennes de fort lessivage et appauvrissement en éléments minéraux. Il présente des tables de grès sableux découpés par des fortes érosions et une foule de collines aux flancs greffés de ravins (INRAP, 2010).

\section{Troupeau}

Le troupeau de porcs Duroc de l'étude est constitué au départ de 10 femelles et 5 mâles de 3 mois importés de France. Ensuite, 145 porcelets se sont ajoutés après la mise en reproduction des parentaux.

\footnotetext{
Alimentation

Elle a été basée sur la distribution de rations provenant d'aliments provendes à raison de $0,5 \mathrm{~kg} /$ porcelets les deux premières semaines après la naissance, $1 \mathrm{~kg}$ à quatre semaines, $1,5 \mathrm{~kg}$ à 6 semaines, $2 \mathrm{~kg}$ à 8 semaines. Entre la 10 ème et la 16 ème semaine les animaux ont reçu $2,5 \mathrm{~kg}$ de ration. L'abreuvement s'est fait ad libitum.
}

\section{Prophylaxie}

Le contrôle sanitaire des animaux s'est effectué régulièrement, ils ont subi un déparasitage interne tous les 3 mois et un autre externe tous les 6 mois.

\section{Evaluation des caractéristiques reproductives}

La détermination de l'âge et le poids de mise en reproduction des mâles et femelles s'est faite à base des consultations des fiches techniques et des mensurations directes. Les poids individuels ont été obtenus en pesant les animaux. L'état corporel des cochettes et des verrats a été évalué selon un système de pointage de 1 à 5 en fonction de l'état d'engraissement ou de l'embonpoint des animaux. Chaque reproducteur est mis en station debout, pour examiner les apophyses des vertèbres et la région costale.

Le touché a permis d'apprécier le gras à la recherche d'une meilleure conformation selon la méthode décrite par Faccenda (2015) et Fabrega (2013). Aussi, l'état des trayons de la femelle a été exploré. Pour accoupler les animaux, le réflexe d'immobilité a été pris en compte.

\section{Croissance pondérale des animaux}

Le suivi de l'évolution pondérale des mâles et femelles s'est fait à travers le Gain Moyen Quotidien, l'Indice de Consommation et les pesées des animaux.

\section{Estimation des productivités numérique et pondérale}

Les productivités numérique(Pn) et pondérale $(\mathrm{Pp})$ du troupeau sont calculées à l'aide des formules suivantes:

\footnotetext{
$\mathrm{Pn}=$

$\frac{\text { NOMBRE MOYEN DES PORCELETS SEVRES PAR TRUIE }}{\text { NOMBRE DE FEMELLES MISE EN REPRODUCTION }}$ an

$\mathrm{Pp}=\frac{\text { POIDS VIF MOYEN DES PORCELETS SEVRES }}{\text { NOMBRE DE FEMELLES MISE EN REPRODUCTION }}$
/an
} 


\section{Estimation du rendement de la carcasse}

Le rendement est une production évaluée par rapport à une unité de mesure, il peut être technique ou commerciale. En ce qui nous concerne, le rendement à l'abattage est le rapport entre le poids de la carcasse pesé le lendemain de l'abattage et le poids vif final, avant la mise à jeun.

Après abattage, on juge la valeur du porc de boucherie sur la carcasse et l'on dispose de deux critères : le rendement et la qualité. Le rendement est donné par la formule suivante (Ognika, 2018; Youssao et al., 2004) :

Rendement moyen $=\frac{\text { poids de la carcasse }}{\text { poids vif }} X 100$

\section{RESULTATS}

\section{Indices de reproduction des truies Duroc}

Les indices de reproduction et les résultats des portées des truies Duroc sont mentionnés dans le Tableau 1. Ils indiquent que le nombre de porcelets par truie et par mise-bas est de 9,5 $\pm 0,6$ têtes et le nombre de porcelets

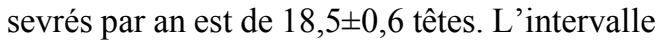
entre mise-bas est de $162,5 \pm 1,2$ jours.

\section{Croissance pondérale des porcelets de race Duroc}

Les porcelets de race Duroc (Tableau 2)

indiquent un poids vif moyen de $1,32 \pm 0,4 \mathrm{~kg}$ à la naissance, $9,99 \pm 0,1 \mathrm{~kg}$ à la 4 ème semaine correspondant au sevrage, $19,8 \pm 1,4 \mathrm{~kg}$ à la $8^{\text {ème }}$ semaine, $33,7 \pm 0,9 \mathrm{~kg}$ la $12^{\text {ème }}$ semaine pour se fixer à $50,4 \pm 6,5 \mathrm{~kg}$, la $16^{\text {ème }}$ semaine.

Les GMQ ont été respectivement de $230 \pm 0,5 \mathrm{~g}$, la 2 ème semaine, $370 \pm 0,3 \mathrm{~g}$, la $4^{\text {ème }}$ semaine, pour atteindre $430 \pm 0,2 \mathrm{~g}$, la $8^{\text {ème }}$ semaine et $650 \pm 0,8 \mathrm{~g}$, la $16^{\text {ème }}$ semaine. L'Indice de consommation des animaux durant les mêmes semaines d'étude ont été respectivement de $1,21 \pm 0,28 ; 2,5 \pm 0,30$; $2,25 \pm 0,31$ et de $2,38 \pm 0,24$.

\section{Corrélations linéaires}

Les corrélations linéaires entre le poids vif, le GMQ et l'IC des porcelets Duroc (Figures 1,2 et 3) sont largement positives. Il ressort une relation étroite entre le poids vif et le GMQ, (Figure 1) si l'on considère toute la période de croissance des animaux $\left(\mathrm{R}^{2}=0\right.$, 6684). Ce qui traduit une bonne prise de poids convertie en muscle. L'indice de consommation n'a pas connu de forte variation durant toute la période de croissance évoluant entre 0,60 et $1,40\left(R^{2}=0,8879\right)$. Ce critère explique la bonne conversion des aliments qui traduit une bonne efficacité alimentaire.

Tableau 1 : Indices de reproduction des truies Duroc.

\begin{tabular}{lll}
\hline Indices & Valeurs & CV \\
\hline Nombre de portée par an & $2,1 \pm 0,7$ & 12 \\
\hline Taille de la portée par truie & $9 \pm 0,8$ & 5,4 \\
\hline Poids de la portée par an $(\mathrm{kg})$ & $25,08, \pm 8$ & 11 \\
\hline Intervalle entre mise-bas(jours) & $162,5 \pm 1,2$ & 3,02 \\
\hline Intervalle sevrage saillie fécondante & $20,2 \pm 5$ & 10 \\
\hline Productivité numérique des truies & 1,2 & 0,4 \\
\hline Nombre de porcelets sevrés/ portée & $9,25 \pm 6$ & 82,22 \\
\hline Age de mise en reproduction ( mois) & $8,3 \pm 0,7$ & 6,2 \\
\hline Mortalité avant sevrage(\%) & $4,12 \pm 0,8$ & 56,32 \\
\hline Mortalité post sevrage $(\%)$ & $3,1 \pm 0,3$ & 72,21 \\
\hline Durée de gestation & $114,1 \pm 1,3$ & 103,42 \\
\hline
\end{tabular}


Tableau 2 : Croissance pondérale des porcelets Duroc.

\begin{tabular}{|c|c|c|c|c|c|c|c|}
\hline $\begin{array}{l}\text { VARIABLES } \\
\text { ADAPTEES }\end{array}$ & S1 & S4 & S8 & S12 & S16 & 20 & 24 \\
\hline POIDS (kg) & $1,32 \pm 04$ & $9,99 \pm 0,1$ & $\begin{array}{l}19,8 \pm \\
1,4 \\
\end{array}$ & $33,7 \pm 0,9$ & $50,4 \pm 6,5$ & $73,4 \pm 3,6$ & $83,7 \pm 1,1$ \\
\hline GMQ(g) & & $370 \pm 0,3$ & $430 \pm 0,1$ & $600 \pm 0,12$ & $650 \pm 0,10$ & $659 \pm 4$ & $665 \pm 5,2$ \\
\hline IC & & 1,21 & 2,5 & 2,25 & 2,38 & 2,46 & 2,81 \\
\hline $\begin{array}{l}\text { POIDS } \\
\text { CARCASSE }(\mathrm{kg})\end{array}$ & ------- & ------ & ------ & ------- & ------ & -------- & $48,6 \pm 0,4$ \\
\hline $\begin{array}{l}\text { RENDEMENT } \\
\text { CARCASSE }(\%)\end{array}$ & ----- & ------- & -------- & ------- & ------- & -------- & 58,06 \\
\hline $\begin{array}{l}\text { VARIABLES DE } \\
\text { REFERENCE }\end{array}$ & S1 & S4 & S8 & S12 & S16 & S20 & S 24 \\
\hline POIDS VIF (kg) & 1,40 & 12 & 25 & $30-40$ & 60,5 & 90 & 100 \\
\hline GMQ(g) & & 400 & 650 & 700 & 750 & 800 & 850 \\
\hline IC & & 1,20 & 1,47 & 1,78 & 2 & 2,45 & 2,45 \\
\hline $\begin{array}{l}\text { POIDS } \\
\text { CARCASSE }(\mathrm{kg})\end{array}$ & ------- & ------ & ------ & ------- & ------ & -------- & 54 \\
\hline $\begin{array}{l}\text { RENDEMENT } \\
\text { CARCASSE }(\%)\end{array}$ & ----- & ------- & -------- & ------- & ------- & -------- & 57 \\
\hline
\end{tabular}

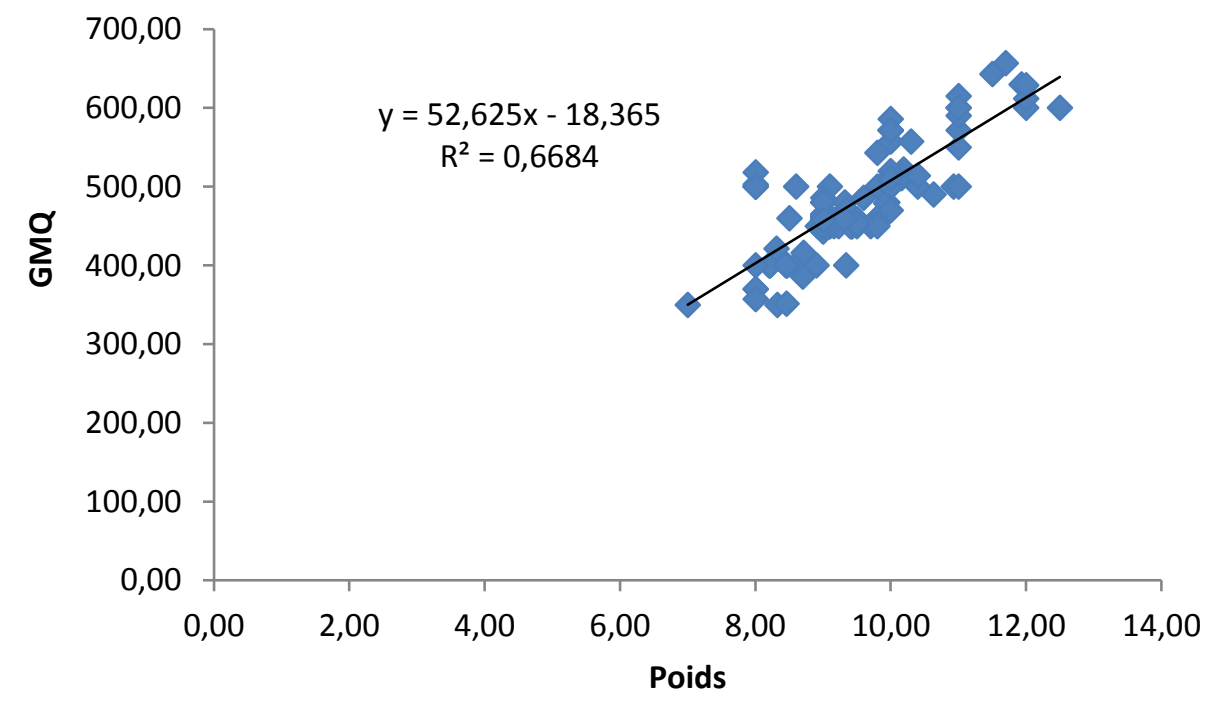

Figure 1 : Corrélation entre le poids vif et le GMQ. 
A. J. OGNIKA et al. /Int. J. Biol. Chem. Sci. 15(5): 1853-1862, 2021

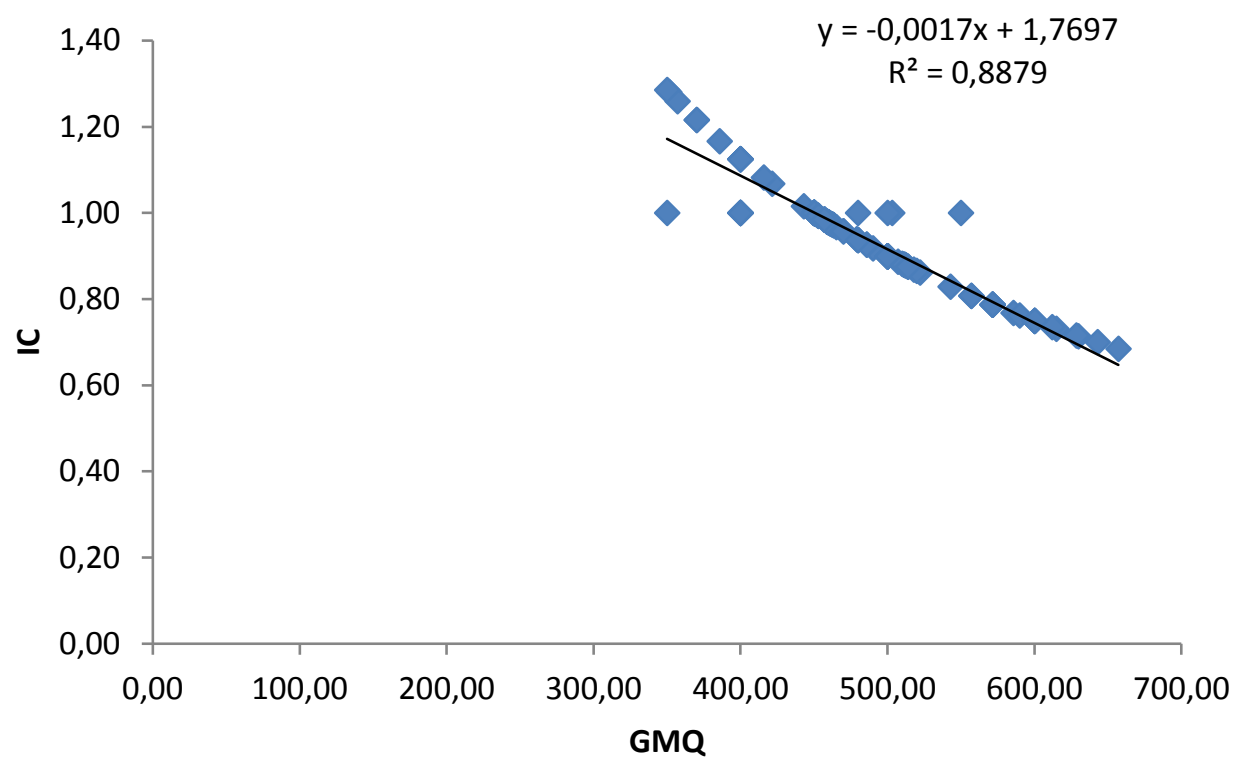

Figure 2 : Corrélation entre le GMQ et l'Indice de Consommation (IC).

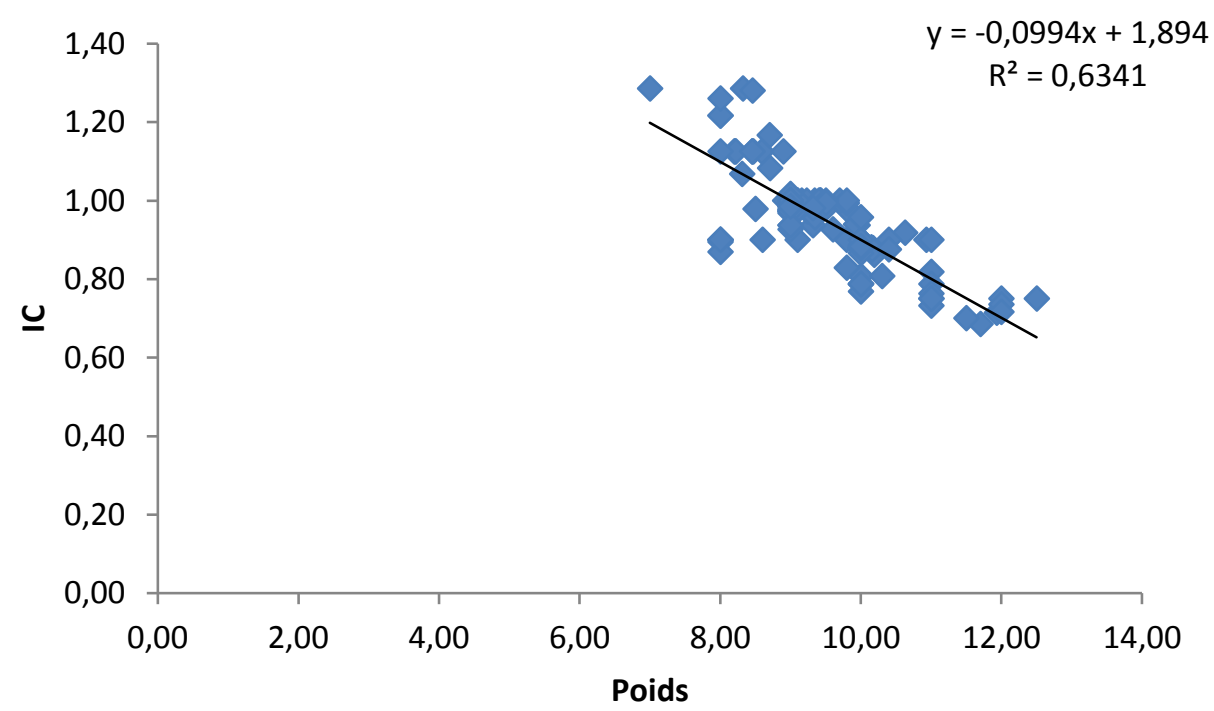

Figure 3 : Corrélation entre le poids et l'IC. 


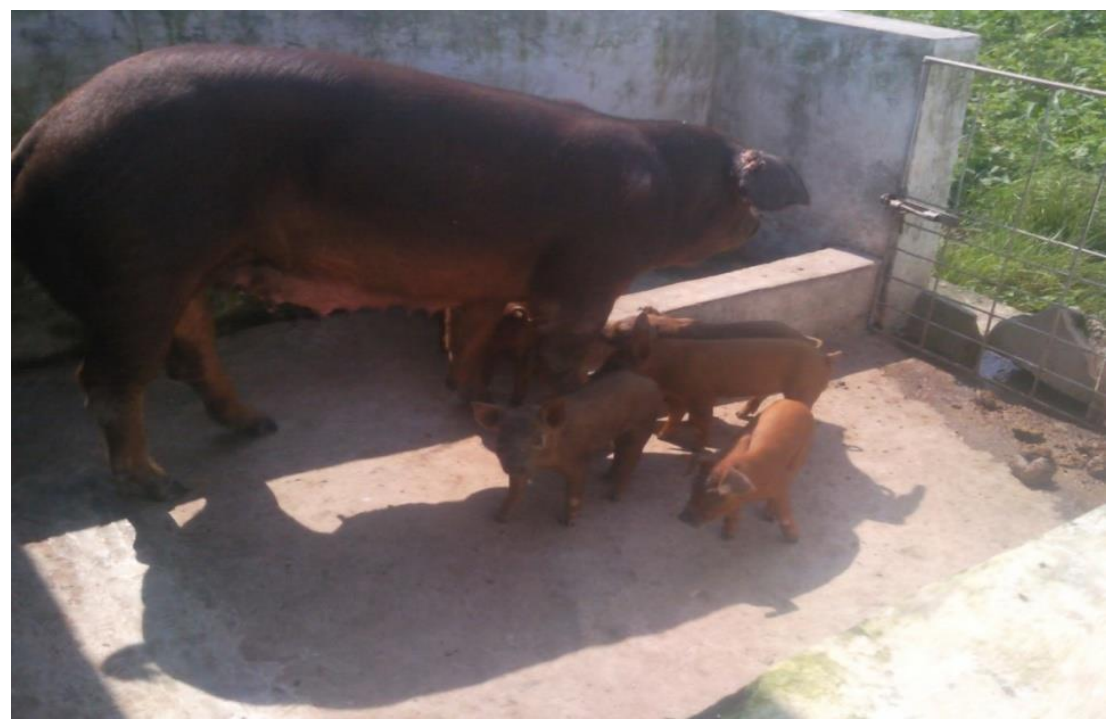

Figure 4 : truie Duroc et ses porcelets allaitants

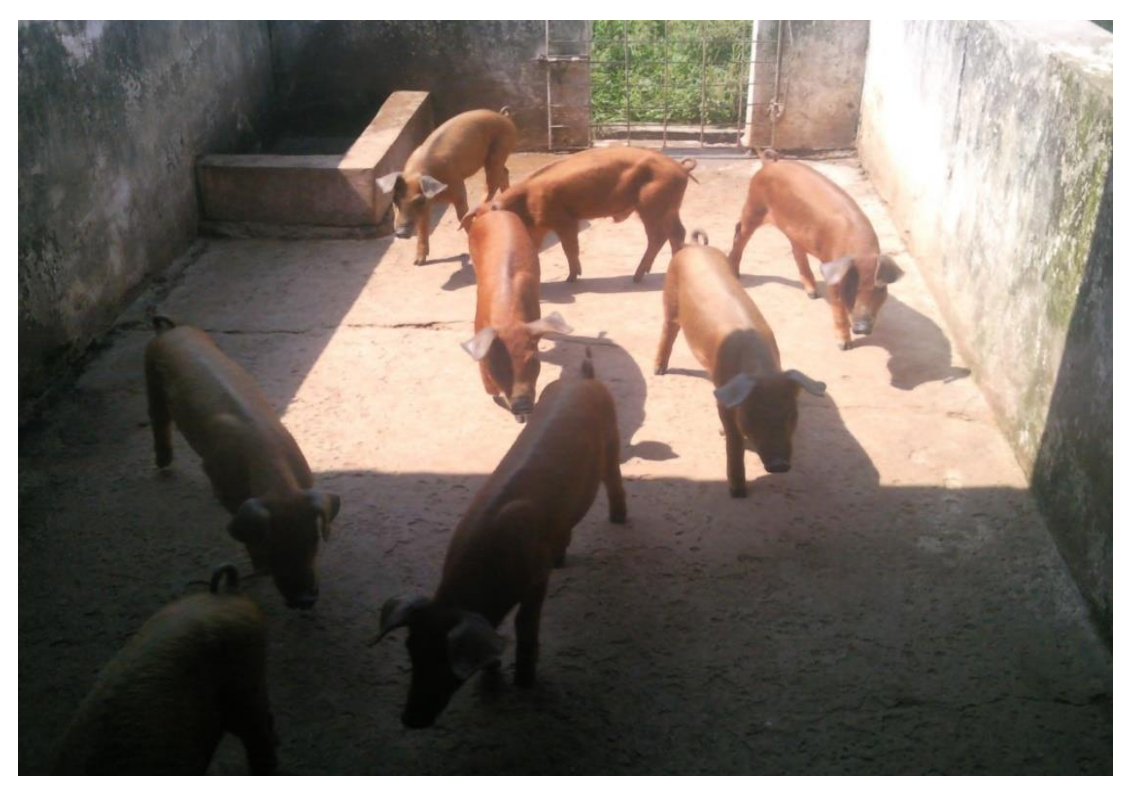

Figure 5 : Porcelets Duroc à l'engrais.

\section{DISCUSSION}

\section{Croissance Pondérale}

Les porcelets Duroc indiquent un poids vif moyen à la naissance de $1,32 \pm 04 \mathrm{~kg}$, une moyenne au-dessus de $1 \mathrm{~kg}$. Cela s'explique sans doute par le potentiel génétique de la race, réputée dans la production des porcelets lourds (Le Cozier et al., 2004 ; Bernard, 2003 ; Berger, 2001). Les porcelets locaux élevés dans les mêmes conditions enregistrent un poids moyen à la naissance de $0,81 \pm 48,9 \mathrm{~kg}$ sans différence significative comparés aux porcelets de la race Duroc.

\section{Poids au sevrage}

Le poids au sevrage des porcelets était examiné à 4 semaines d'âge et il était de $9,99 \pm 0,1 \mathrm{~kg}$. Ce poids, enregistré par les animaux Duroc au moment du sevrage est certainement lié à l'effet positif de la race sur 
les performances de croissance. Il est comparable au poids obtenu avec les animaux de la race Large White de même âge $(9 \pm 0,14)$, élevés dans les mêmes conditions sans différence significative (Ognika et al., 2016).

Cependant, le poids des animaux de la race Duroc est supérieur à celui du porcelet local $(5,2 \pm 3,27 \mathrm{~kg})$, qui reste largement en dessous avec une différence significative. La prise lente de poids chez le porcelet local et le manque d'importantes réserves corporelles depuis la naissance de celui-ci peuvent expliquer la différence de poids.

\section{Rendement carcasse}

Le poids à l'abattage obtenu à 6 mois d'âge était de $83,7 \pm 1,1 \quad \mathrm{~kg}$. Le potentiel génétique de la race peut expliquer cette importante prise de poids (Huart, 2009), qui demeure inférieure à celle de la race Large White $(87,3 \pm 4,2 \mathrm{~kg})$, élevées dans la même ferme et dans les mêmes conditions, sans être significativement différente (Ognika, 2018). Ce poids reste cependant supérieur au poids des porcs locaux $(45,7 \pm 6,42 \mathrm{~kg})$, avec une différence significative. De nombreux travaux ont déjà souligné le faible potentiel de croissance des races locales par rapport aux races améliorées (Bastianelli et al., 2009; Youssao et al., 2009 ; Texier, 1984).

Quant au rendement carcasse, il indique $(58,5 \pm 6,7 \%)$; une moyenne supérieure au seuil acceptable de $50 \%$, ce qui prouve la possibilité d'obtenir de très bons rendements de carcasse de la race Duroc à l'abattage au Congo. Ce résultat est comparable à celui obtenue par les chercheurs de l'Ifip (2018), sur la race Duroc avec un rendement moyen de $56 \%$. Toutefois, il reste supérieur et diffère significativement du rendement de la race locale $(48 \pm 3,5 \%)$. Ainsi, le rendement carcasse obtenu pendant l'étude comme l'ont affirmé Youssao et al. (2004), peut servir comme indice de productivité avec des répercussions sur le rendement en viande.

\section{Productivités numérique et pondérale}

La productivité numérique des truies Duroc a été de 1,2, le nombre de porcelets par truie et par mise-bas de 9,5 $\pm 0,6$ têtes, le nombre de porcelets sevrés par an de 18,5 $\pm 0,6$ têtes. Ce niveau de productivité marque la bonne évolution du troupeau et révèle l'importance du type génétique des animaux en élevage de porcs (Labroue et al., 2000). Ce résultat est inférieur à celui obtenu avec les animaux de la race Large White dans les mêmes conditions d'élevage (Ognika, 2018). Wavreille et al. (2001) ont également obtenu les meilleures performances zootechniques avec la race Large White comparée aux races Landrace, Duroc et piétrain.

\section{Indices de reproduction des truies}

L'évaluation de l'âge à la puberté et de mise en reproduction s'est faite à travers les fiches techniques et a concerné les 10 cochettes de départ présentes sur l'élevage. La détection des chaleurs est faite deux fois par jour $(8 \mathrm{~h}$ et $17 \mathrm{~h})$, avec un verrat présenté devant les truies.

La taille de la portée était de $(9 \pm 0,8)$ et le nombre de porcelets nés vivants de $(9,25 \pm$ 6). Le suivi zootechnique et zoosanitaire accordé aux porcelets peut expliquer ce résultat. Labroue et al. (2000) par contre ont obtenu des différences significatives après observations de ces deux paramètres sur les races Boyeux et Gascon. Ces résultats font aussi apparaître une diminution de la taille de portée comparés à l'étude réalisée sur les porcs de la race Large White (15 porcelets) de la même ferme élevés dans les mêmes conditions (Ognika et al., 2016) Cette tendance à la baisse est inexpliquée puisque la moyenne de la race en termes de portée selon les études de Huart (2013) sur le comportement de la race en Afrique se situe à 12 porcelets par mise-bas. D'autres expériences seront nécessaires pour continuer à suivre l'évolution de la race au Congo.

Le poids de la portée était de 11,6 $\pm 0,4$ $\mathrm{kg}$, l'explication réside sans nul doute dans l'objectif même de la race. Il s'agit d'une race à viande spécialisée dans la production de porcelets lourds. On peut dire que les performances calculées reflètent bien le niveau génétique de la race au Congo. Ce résultat est supérieur et diffère significativement de celui 
obtenu avec la race locale 4,86 $\pm 48,9 \mathrm{~kg}$ élevée dans la même localité (Ognika, 2018).

\section{Conclusion}

La présente étude apporte de la matière aux problèmes de reproduction et de croissance de la race Duroc au Congo. En partant des saillies, les observations ont porté sur la gestation, les pesées des porcelets à la naissance, la courbe de croissance, le rendement de la carcasse et les indices reproductifs des porcs Duroc. Les études ont été menées dans le but de mieux appréhender le niveau d'adaptation de la race au Congo à travers les performances de production et de reproduction en vue de l'incorporer au schéma d'amélioration du porc local en République du Congo.

\section{CONFLIT D'INTERETS}

Les auteurs déclarent qu'ils n'ont pas de conflit d'intérêts.

\section{CONTRIBUTIONS DES AUTEURS}

Tous les auteurs ont participé à l'étude sur le terrain avec AJO comme investigateur principal. Ils ont lu et validé le présent article.

\section{REMERCIEMENTS}

Les auteurs de ce document remercient l'ingénieur Moutinga et tout le personnel du CVTE Kombé de leur collaboration et pour avoir autorisé cette étude.

\section{REFERENCES}

Accodji JMM, Fiogbe ED, Gangbazo. 2009. Esssai de valorisation d'Azolla (Azolla microphylla Kaulf) dans la production porcine en zone humide. Int. J. Biol. Chem. Sci., 3(5): 890-898. DOI: 10.4314/ijbcs.v3i5.51054

Bastianelli D, Derail L, Klotz S. 2009. L'élevage de porc. Journal de Recherche Porcine, 32: 721-725. DOI : https://doi.org/10.20870.

Berger F. 2001. Les clefs d'une insémination réussie. Revue Porc Magazine, 340: 8186. DOI : https://porc magazine 340.

Bernard G. 2003. Nouvelle technologie en matière d'insémination artificielle. Revue
Porc Québec, 437: 11-14. DOI : https://porc Québec 437.

Direction Générale de l'élevage (DGE). 2014. Rapport d'activité de la Direction Générale de l'Elevage, année 2013. Brazzaville Congo, 50p.

Fabrega. 2013. Evaluation de l'état corporel des porcs. Texte et illustrations réalisés d'après Condition Scoring of Pigs PB 3480 Defra 1998. Crown copyright material is reproduced with the permission of the controller of HMSO and queen's Printer for Scotland. DOI: http://vigiferme.org/index.php.

Faccenda M. 2015. Etat d'engraissement des truies, http://vigiferme.org/index.php, 6p.

Institut français international du porc(IFIP), 2010. Maitrise de la croissance des porcs par l'indice de consommation. Fiche 56. DOI : http://www.ifip.asso.fr/resultats. Consulté le 11 février

Institut français international du porc (IFIP), 2018. Caractéristiques de la race Duroc. DOI : http:/www.ifip.asso.fr/resultats.

Huart A. 2013. Comparaison des races Large White, Piétrain et Duroc pour les performances de croissance et de reproduction. ECO-CONGO. Agriculture et Entreprenariat, Centre Agronomique et Vétérinaire. (1) n`2. 20-23.

Institut National de Recherche et d'Action Pédagogique (INRAP), 2010. Notions de Géographie, édition revue et corrigée. Edicef 58, Vanves (France), $121 \mathrm{p}$.

Kimsé M, Soro D, Bléyéré JN, Fantodji A. 2013. Apport d'un fourrage vert tropical, Centrosema pubescens, en complément au granulé : effet sur les performances de croissance et sanitaire du lapin (Oryctolagus cuniculus). Int. J. Biol. Chem. Sci., 7(3) : 1234-1242. DOI: http://dx.doi.org/10.4314/ijbcs.v7i3.29

Labroue F, Goumy S, Gruand J, Mouro, Neelz V, Legault C. 2000. Comparaison au large White de quatre races locales porcines françaises pour les performances de croissance, de carcasse et de qualité de la viande. Journées Recherche Porcine en France, 32: 403-411. DOI: https://journéé de recherche porcine 29. 
Le Cozier Y, Pichodo H, Guyomarch C, Pellois $\mathrm{N}$, Quiniou N, Louveau I, Lebret, Lefaucher L, Gondre T. 2004. Influence du poids individuel et de la taille de la portée à la naissance sur la survie du porcelet, ses performances de croissance et d'abattage et la qualité de la viande. Journées recherche porcine, 36, pp : 443-450. DOI : https://journéé de recherche porcine 36 .

Mopoundza P, Missoko Mabeki R, Angandza SG, Mbou AS. 2019. Prévalence de la cysticercose porcine à Taenia solium (Cysticercus cellulosae) chez les porcs dans l'aire d'abattage de Kinsoundi à Brazzaville. Int. J. Biol. Chem. Sci., 13(3) : 1396-1410. DOI: https://dx.doi.org/10.4314/ijbcs.v13i3.15

Ognika AJ, Mopoundza P, Okandza Y, Akouango P. 2016. Adaptation productive et reproductive des porcs Large White élevés en race à Brazzaville République du Congo. Journal of Animal \& Plant Science, 30 (1) : 4727-4735.

Ognika A, Missoko R, Mopoundza P, Akouango P. 2016. Dynamique des élevages et caractéristiques des producteurs de porcs de Brazzaville et ses environs. Journal of Animal \& Plant Science, 29 (1): 4426-4536.

Ognika AJ. 2018. Contribution des races Large White, Landrace et Duroc à la dynamique de productivité du porc local à Brazzaville et sa périphérie (République du Congo). Thèse de doctorat unique, Université Marien Ngouabi 166 p.
Texier C, Luquet M, Boudy A. 1984. Comparaison des performances d'engraissement et des qualités de carcasse des porcs de races locales Normande, Basque et Bayeux. Journées Recherche Porcine en France, 28: 42-46. DOI : https://journéé de recherche porcine 28.

Warveille J, Faes T, Sindic M, Claustriaux JJ, Lognais G, Bartiaux N. 20001. Comparaison des performances zootechniques et étude de la qualité de la viande de porc landrace francais, Large White et pietrain engraissés en porcherie. Journées de la Recherche Porcine, 34 : 23-29. DOI : https:// journéé de recherche porcine 34 .

Youssao A, Koutinhouin G, Kpodekon A, Yacoubou, Bonou A, Adiakpa A, Ahounou, Taiwd, 2009. Amélioration génétique des performances zootechniques du porc local du Benin par croisement avec le Large White. Int. J. Biol. Chem. Sci., 3(4): 653-662. DOI: 10.4314/ijbcs.v3i4.47158

Youssao A, Mouroi J, Gbangbche A, Adehan R, Akoutey A, Edenakpo. 2004. Influence $\mathrm{du}$ régime alimentaire sur les performances de croissance et les caractéristiques de la carcasse du porc de race locale au Benin. Revue Africaine de Santé et de Productions Animales (RASPA), 2(1) : 31-36. 